\title{
MS03-P10 | Sec-Saxs Analysis Of Oligomeric States Of Human Nkr-P1 With Its LIGAND LLT1 IN SOLUTION
}

Skalova, Tereza (Institute of Biotechnology CAS, v.v.i., Vestec, CZE); Blaha, Jan (Department of Biochemistry, Faculty of Science, Charles University, Prague, CZE); Stransky, Jan (Institute of Biotechnology, The Czech Academy of Sciences, v.v.i., Vestec, CZE); Koval, Tomáš (Institute of Biotechnology of the Czech Academy of Sciences, Vestec, CZE); Duskova, Jarmila (Institute of Biotechnology, The Czech Academy of Sciences, v.v.i., Vestec, CZE); Skorepa, Ondrej (Department of Biochemistry, Faculty of Science, Charles University, Prague, CZE); Kalousková, Barbora (Department of Biochemistry, Faculty of Science, Charles University, Prague, CZE); Pazicky, Samuel (Department of Biochemistry, Faculty of Science, Charles University, Prague, CZE); Vanek, Ondrej (Department of Biochemistry, Faculty of Science, Charles University, Prague, CZE); Dohnálek, Jan (Institute of Biotechnology of the Czech Academy of Sciences, Vestec, CZE)

Natural killer (NK) cells are a type of lymphocytes able to kill tumour and virally infected cells. Human NKR-P1 is one of the plenty of receptors anchored in the membrane of the cell and LLT1 is its ligand on a partner cell. Both NKR-P1 and LLT1 have extracellular part with C-type lectin like fold.

The extracellular domains of NKR-P1 and LLT1 have been expressed and characterised.1,2 The crystal structures of LLT1 oligomers have been published3 and structures of NKR-P1 and NKR-P1:LLT1 have been deposited. We discovered that NKR-P1 and LLT1 form a chain in the crystal structure of the NKR-P1:LLT1 complex. In order to study the form of the interaction of NKR-P1 with LLT1 in solution, we have performed small angle X-ray scattering coupled with size exclusion chromatography (SEC-SAXS). The SEC-SAXS intensity curve shows two distinct peaks, the smaller one corresponding to higher oligomers and the larger one corresponding basically to the NKR-P1:LLT1 complex. However, deeper analysis showed that in fact, each point of the SEC-SAXS curve corresponded to a combination of several oligomeric states.

[1] Bláha, J. et al (2015). Protein Expr. Purif. 109, 7-13.

[2] Bláha, J. et al (2017). Protein Expr. Purif. 140, 36-43.

[3] Skálová, T. et al (2015). Acta Cryst. D71, 578-591.

This study was supported by BIOCEV (ERDF CZ.1.05/1.1.00/02.0109), CIISB4HEALTH (ERDF

CZ.02.1.01/0.0/0.0/16_013/0001776), Czech Science Foundation (15-15181S and 18-10687S), MEYS of the Czech Republic (LTC17065 within the COST Action CA15126), Charles University (SVV260427/2018, GAUK 161216), and Instruct (R\&D pilot scheme APPID 56 and 286). 